\title{
Characteristics of occlusion in primary dentition of preschool children of Udaipur, India
}

\author{
Sapna Hegde ${ }^{1}$ \\ Sunil Panwar ${ }^{1}$ \\ Dinesh Rao Bolar ${ }^{1}$ \\ Mitesh Bharat Sanghavi ${ }^{1}$
}

\section{ABSTRACT}

Objective: The present study aimed to study the occlusion characteristics of the primary dentition in a group of 3-5 year-old Indian children and the differences with age in the same group.

Methods: The study was a cross-sectional survey based on examination of the primary dentition of 200 preschool children aged 3-5 years who were selected from nursery schools in Udaipur, India, using a stratified random sampling technique. The study group was assessed for the several occlusal parameters, which included primary molar and canine relationship, degree of overjet and overbite, anterior and posterior crossbite, and the presence or absence of physiologic spaces and crowding.

Results: Flush terminal plane was more common at 3-4 years of age, mesial step at 4-5 years, and class I canine relationship in both age groups. The prevalence of overjet less than $1 \mathrm{~mm}$ and overjet exceeding $1 \mathrm{~mm}$ was almost comparable in both groups. Overbite with less than $30 \%$ overlap was most frequently observed in both age groups, with 30-60\% overlap following closely behind. Physiologic and primate spacing were observed in less than half of the children examined, while a small percentage displayed the presence of either single-segment or two-segment crowding.

Conclusion: The present study provided insight into the state of dentition, occlusal pattern, spacing, and crowding in the primary dentition of Indian children of Udaipur. (Eur J Dent 2012;6:51-55)

Key Words: Primary occlusion, occlusion, occlusion characteristics, primary dentition

\section{INTRODUCTION}

Occlusion in the primary dentition plays a significant role in determining the space for and occlusion in the succeeding permanent dentition. ${ }^{1,2}$

1 Department of Paediatric Dentistry, Pacific Dental College \& Hospital, Debari, Udaipur - 313024, Rajasthan, INDIA

Corresponding author: Dr. Sapna Hegde, Department of Paediatric Dentistry, Pacific Dental College \& Hospital, Debari, Udaipur - 313024, Rajasthan, INDIA Phone: +919828144002 Fax: +91 2942491508 E-mail: drsapnahegdeđyahoo.co.in
The characteristic set of features of this dentition to a large extent lays the foundation for proper eruption and alignment of the succeeding dentition. Based on the observation of these key features of occlusion in the child's dentoalveolar system during the formative years, the characteristics of the permanent dentition occlusion can be predicted very well.

Although the occlusion of the permanent dentition is largely influenced by the framework provided by the preceding primary dentition, its fea- 
tures vary among different populations and ethnic groups. ${ }^{3-13}$ Contradictory findings documented in the literature worldwide compounded by inadequate information on the primary dentition of Indian children, provided the motivation for this study, which was carried out with the aims of al assessing the occlusion characteristics of the primary dentition in a group of 3-5 year-old Indian children in Udaipur, Rajasthan, and b) assessing the differences with age, in the same group.

\section{MATERIALS AND METHODS}

Our cross-sectional survey was based on examination of the primary dentition of 200 preschool children aged 3-5 years from Udaipur, India. The sample was selected from nursery schools using a stratified random sampling technique and the age of each child obtained from school records.

Healthy preschool children aged 3-5 years, devoid of systemic disease, physical or mental abnormality, any developmental defects, or carious teeth, were included in the study. The children were divided into Group A-those between 3 and 4 years of age-and Group B-those between 4 and 5 years of age.

Examination was performed by a single examiner with the aid of penlight, mouth mirror, and metal millimeter ruler. Occlusal assessment was carried out with the teeth in centric occlusion and in children who had a complete primary dentition without any erupted permanent teeth.

The following parameters were recorded:

1. Primary molar relationship (terminal plane): the relationship of the maxillary and mandibular second primary molars in the vertical plane.

a. Flush terminal plane: the distal surfaces of maxillary and mandibular primary second molars lie in the same vertical plane.

b. Distal-step: the distal surface of mandibular primary second molar is distal to that of the primary maxillary second molar.

c. Mesial-step: the distal surface of mandibular primary second molar is mesial to that of the maxillary primary second molar.

2. Primary canine relationship:

a. Class I: the tip of the maxillary canine is in the same vertical plane as the distal surface of the mandibular canine.

b. Class II: the tip of the maxillary primary canine tooth is mesial to the distal surface of the mandibular primary canine. c. Class III: the tip of the maxillary canine is distal to the distal surface of the mandibular primary canine.

Decisions regarding molar and canine relationship were made on the basis of bilateral occlusion. In case of distal step or mesial step relationship on one side and flush terminal plane on the other, the decision was made in favor of flush terminal plane.

The canine relationship was recorded in favor of class I, if it was class I on one side and class II or class III on the other. In the event that the child had class II canine relation on one side and class III on the other side, the case was excluded from canine relationship.

3. The degree of overbite was graded according to coverage of mandibular incisor by the most protruded fully erupted maxillary incisor.

a. Normal coverage of up to half the mandibular incisor by the maxillary incisors.

b. Increased coverage of more than half the mandibular incisors by maxillary incisors.

c. Edge-to-edge relation.

d. Anterior open bite-negative overlap in the vertical plane.

4. The degree of overjet was measured from the palatal surface of the mesial corner of the most protruded fully erupted maxillary incisor to the labial surface of the corresponding mandibular incisor and was recorded in millimeters.

5. Anterior cross-bite was recorded when one or more maxillary incisors and canines occluded lingual to the mandibular incisors.

6. Posterior cross-bite was recorded when one or more maxillary primary canines or molars occluded lingual to the buccal cusps of the opposing mandibular teeth.

7. Scissor-bite was recorded when one or more maxillary primary molars occluded buccal to the buccal surface of the corresponding mandibular molars.

8. The presence or absence of physiologic spaces between primary teeth was noted, as was the presence or absence of primate spaces (spaces mesial to maxillary canine and distal to mandibular primary canine). ${ }^{11}$

9. The presence of crowding was considered as either single-segment (in one arch only) or twosegment (in both arches). ${ }^{12}$

To ensure intra-examiner reliability, the same examiner examined 30 children on two occasions 
at least one week apart. ${ }^{11}$ The results obtained were almost the same for all parameters (Kappa Value $=.89-.94)$.

The results were tabulated and analyzed using the Statistical Package for Social Sciences (SPSS) software version-15 for Windows. Comparisons between groups were carried out using the Pearson's Chi-square test. For all tests, a p-value of .05 or less was considered to be of statistical significance.

\section{RESULTS}

The present study assessed the occlusal characteristics of the primary dentition of 200 children aged 3 to 5 years. The results are presented in Table 1.

The study revealed that the most common molar relationship in the 3-4 year age group (Group A) was the flush terminal plane (52\%), while in the 4-5 year age group (Group B), mesial step was most commonly seen (54\%). In Group A, mesial step and distal step were seen in $46 \%$ and $2 \%$ of the children respectively. In Group B, $46 \%$ of the children were observed to have a flush terminal plane. Distal step was not observed in any of the children in this age group. In both groups, distal step molar relation was found to decrease and mesial step to increase with increasing age. The difference between groups was not significant statistically.

With respect to canine relationship, class I represented $78 \%$ of the children in Group A, followed by class II, which was observed in $22 \%$. Class III and cusp-to-cusp relationships were not found in any of the children in this group. In Group B, $82 \%$ of children were found to have a class I, and $18 \%$, a class II canine relationship. Again, none of the children examined showed class III or cusp-tocusp relationships, and there was no statistically significant difference between groups.

In Group A, 45\% of the children exhibited overjet less than $1 \mathrm{~mm}, 47 \%$ had overjet more than 1 $\mathrm{mm}$, and $8 \%$ had overjet more than $3 \mathrm{~mm}$. In Group $\mathrm{B}, 43 \%$ exhibited overjet less than $1 \mathrm{~mm}, 48 \%$ had overjet more than $1 \mathrm{~mm}$, and $9 \%$ had overjet exceeding $3 \mathrm{~mm}$.

The majority of the children (46\%) in Group A had overbite with less than 30\% overlap, 39\% showed $30-60 \%$ overlap, and $14 \%$ demonstrated overlap greater than 60\%. Similarly, in Group
B, $44 \%$ had overbite with less than $30 \%$ overlap, while $43 \%$ exhibited $30-60 \%$ overlap, and only $13 \%$ exhibited overlap greater than $60 \%$. Reverse bite (anterior crossbite) was observed in only $1 \%$ of the children in Group A. Two percent of children in Group A and 7\% in Group B exhibited an edgeto-edge incisal relationship. Posterior crossbite was not observed in either group. No statistically significant differences in incisal relationship were observed between Groups A and B.

Physiologic spacing was observed in the maxillary arch of $41 \%$ and mandibular arch of $40 \%$ of children in Group A, while in Group B 57\% and $60 \%$ had physiologic spacing in the maxillary and mandibular arch respectively. Primate spaces were present in the maxillary arch of $56.5 \%$ and mandibular arch of $38 \%$ of the children examined. Twenty-nine percent in Group A and 24\% in Group $B$ exhibited single-segment crowding. Twenty-four percent of children in Group A and 12\% in Group B showed the presence of two-segment crowding. The differences between the two groups were statistically significant for maxillary and mandibular, physiologic and primate spacing, and crowding.

\section{DISCUSSION}

Numerous epidemiological studies ${ }^{3-13}$ have been carried out to document the characteristics of the primary dentition observed in various populations. These studies are important because they often reflect the need for interceptive and corrective orthodontic treatment. The lack of such statistical data of the Indian population in Udaipur prompted us to undertake this study.

The most common molar relationship observed in the 3-4 year group (Group A) was the flush terminal plane (52\%), while in Group B (4-5 year group), mesial step was most commonly seen (54\%). In Group A, mesial step and distal step were seen in $46 \%$ and $2 \%$ of the children examined respectively. In Group B, $46 \%$ of the children showed a flush terminal plane, while distal step was observed in none. In both groups, distal step molar relation was found to decrease and mesial step to increase with increasing age. These findings are concurrent with those of a study of a Saudi Arabian population ${ }^{11}$ and almost similar to that of a study of a Jordanian population, ${ }^{13}$ in which mesial step was most commonly observed and distal step molar relationship was least common. 
Eighty percent of the study group had primary canines in class I relationship and $20 \%$ in class II canine relationship. No class III or cusp-to-cusp canine relationship was observed. The prevalence of class II relationship was higher in 3 year olds than in the 5 year age group, whereas that of class III was lower, but neither difference was statistically significant. These results concur with those reported by studies on Saudi Arabian ${ }^{11}$ and Jordanian children. ${ }^{13}$

Out of the 200 children surveyed, $47.5 \%$ had overjet less than $1 \mathrm{~mm}, 44 \%$ more than $1 \mathrm{~mm}$, while $8.5 \%$ showed more than $3 \mathrm{~mm}$ overjet, consistent with Saudi Arabian ${ }^{11}$ and other Indian studies. $^{4}$

Forty-five percent of the children had overbite with less than $30 \%$ overlap, $41 \%$ showed $30-60 \%$ overlap, and $14 \%$ had more than $60 \%$ overlap. Anterior open bite was not observed in any of the children, which was contradictory to the findings reported for black African (5\%), ${ }^{6}$ Finnish (11\%), ${ }^{8}$ Saudi Arabian (8-11\%), ${ }^{11}$ Nigerian (5.3\%), ${ }^{12}$ and Jordanian $(5.7 \%)^{13}$ children.

Anterior crossbite relationship was observed only in $0.5 \%$ children, and edge-to-edge bite in $4.5 \%$ of the children. These results are in accor- dance with the results of previous studies on English $^{3}$ and Saudi Arabian ${ }^{11}$ populations, but lower than that reported in Finnish, African-American, ${ }^{10}$ and Jordanian ${ }^{13}$ populations.

Posterior crossbite was not observed in our study population compared to the findings in Finnish (13\%), ${ }^{8}$ Saudi Arabian (4\%), ${ }^{11}$ Nigerian (4.8\%), ${ }^{12}$ and Jordanian $(7 \%)^{13}$ children.

Forty-one percent and $40 \%$ of the children in Group A showed the presence of physiologic spaces in the maxillary and mandibular arch respectively, which is lower than that reported in Jordanian children, ${ }^{13}$ while in Group B $57 \%$ and $60 \%$ had physiologic spacing in the maxillary and mandibular arch respectively, which is similar to that observed in Jordanian children. ${ }^{13}$

In the present study, primate spaces were present in $56.5 \%$ children in the maxillary arch and $38 \%$ of the children in the mandibular arch. This is a lower prevalence than that reported by other studies, including one on Jordanian children. 3,12,13

In the present study, of the children examined, $26.5 \%$ exhibited single segment crowding and $19 \%$, two-segment crowding, which is lower than that reported in Nigerian, ${ }^{12}$ Jordanian, ${ }^{13}$ and British children. ${ }^{3}$

Table 1. Group-wise distribution of antero-posterior molar relationship (terminal plane)

\begin{tabular}{|c|c|c|c|c|}
\hline Occlusal parameter & Category/Arch & 3-to-4 years & 4-to- 5 years & Total \\
\hline \multirow{3}{*}{ Terminal plane } & Flush & $52 \%$ & $46 \%$ & $49 \%$ \\
\hline & Mesial-step & $46 \%$ & $54 \%$ & $50 \%$ \\
\hline & Distal-step & $2 \%$ & $0 \%$ & $1 \%$ \\
\hline \multirow{3}{*}{ Canine relationship } & Class I & $78 \%$ & $82 \%$ & $80 \%$ \\
\hline & Class II & $22 \%$ & $18 \%$ & $20 \%$ \\
\hline & Class III & $0 \%$ & $0 \%$ & $0 \%$ \\
\hline \multirow{3}{*}{ Overjet } & $<1 \mathrm{~mm}$ & $45 \%$ & $43 \%$ & $44 \%$ \\
\hline & $1 \mathrm{~mm}-3 \mathrm{~mm}$ & $47 \%$ & $48 \%$ & $47.5 \%$ \\
\hline & $>3 \mathrm{~mm}$ & $8 \%$ & $9 \%$ & $8.5 \%$ \\
\hline \multirow{3}{*}{ Overbite } & $<30 \%$ overlap & $46 \%$ & $44 \%$ & $45 \%$ \\
\hline & $30-60 \%$ overlap & $39 \%$ & $43 \%$ & $41 \%$ \\
\hline & $>60 \%$ overlap & $15 \%$ & $13 \%$ & $14 \%$ \\
\hline \multirow[b]{2}{*}{ Crossbite } & Anterior & $1 \%$ & $0 \%$ & $0.5 \%$ \\
\hline & Posterior & $0 \%$ & $0 \%$ & $0 \%$ \\
\hline \multirow[b]{2}{*}{ Physiological space } & Maxilla & $41 \%$ & $57 \%$ & $49 \%$ \\
\hline & Mandible & $40 \%$ & $60 \%$ & $50 \%$ \\
\hline \multirow[b]{2}{*}{ Primate space } & Maxilla & $46 \%$ & $67 \%$ & $56.5 \%$ \\
\hline & Mandible & $28 \%$ & $48 \%$ & $38 \%$ \\
\hline \multirow{3}{*}{ Crowding } & Absent & $47 \%$ & $64 \%$ & $55.5 \%$ \\
\hline & One-segment & $29 \%$ & $24 \%$ & $26.5 \%$ \\
\hline & Two-segment & $24 \%$ & $12 \%$ & $18 \%$ \\
\hline
\end{tabular}


The present study provided insight into the state of dentition, occlusal pattern, and spacing and crowding in the primary dentition of Indian children of Udaipur. Although the results suggest a low prevalence of malocclusion in the primary dentition of this population, further studies on a larger sample of the same population are warranted, as are studies on children from other states of India.

\section{CONCLUSION}

The following conclusions could be drawn from the present study:

a) Flush terminal plane was more common at 3-4 years of age, mesial step at 4-5 years, and class I canine relationship in both age groups.

b) The prevalence of overjet less than $1 \mathrm{~mm}$ and overjet exceeding $1 \mathrm{~mm}$ was almost comparable in both groups.

c) Overbite with less than 30\% overlap was more frequently observed in both age groups, with 30-60\% overlap following closely behind.

d) Physiologic and primate spacing were observed more frequently in the 4-5 year group, while two-segment crowding was more common in the 3-4 year group.

The present study provides hitherto unavailable data pertaining to the state of dentition, occlusal pattern and spacing, and crowding in the primary dentition of a group of Indian children of Udaipur. This, together with data from studies conducted on other Indian populations, can contribute to a data bank that can be compiled and used for comparison between Indian populations to arrive at a method of predicting any future malocclusion in the permanent dentition.

\section{REFERENCES}

1. Infante PF. An epidemiologic study of deciduous molar relations in preschool children. J Dent Res 1975;54:723-727.

2. Foster TD, Grundy MC. Occlusal changes from primary to permanent dentition. Br Dent Orthod 1986;13:187-193.

3. Foster TD, Hamilton MC. Occlusion in the primary dentition, study of children at $21 / 2$ to 3 years of age. Br Dent $J$ 1969;126:76-79.

4. Nanda RS, Khan I, Anand R. Age changes in the occlusal pattern of deciduous dentition. J Dent Res 1973;52:221-224.

5. Kohler L, Hoslt K. Malocclusion and sucking habit of 4-years-old children. Acta Paediatr Scand 1973;62:373-379.
6. Infante PF. Malocclusion in deciduous dentition in white, black and Apache Indian children. Angle Orthod 1975;45:213-218.

7. Ravn JJ. Longitudinal study of occlusion in the primary dentition in 3-7 year old children. Scand J Dent Res 1980;88:165170.

8. Kerosuo H. Occlusion in primary and early mixed dentition in a group of Tanzanian and Finnish children. J Dent Child 1990;57:293-298.

9. Kritsineli M, Shim Y. Malocclusion, body posture, and temporomandibular disorder in children with primary and mixed dentition. J Clin Pediatr Dent 1992;16:86-93.

10. Jones ML, Murino AP, Bowden TA. Evaluation of occlusion, trauma and dental anomalies in African-American children of metropolitan Headstart programmes. J Clin Pediatr Dent 1993;18:51-54.

11. Farsi MA and Salama FS. Characteristics of primary dentition in group of Saudi children. Int $J$ Paediatr Dent $1996 ; 6: 253-259$

12. Otuyemi OD, Sote EO, Isiekwe MC, Jones SP. Occlusal relationship and spacing or crowding of teeth in the dentitions of 3-4 years-old Nigerian children. Int J Paediatr Dent 1997;7:155-160.

13. Abu Alhaija ESJ, Qudeimat MA. Occlusion and tooth/arch dimension in primary dentition of preschool Jordanian children. Int J Paediatr Dent 2003;13:230-239. 